Review

\title{
Pathophysiological mechanisms of cardiotoxicity in chemotherapeutic agents
}

\author{
Aleksey M. Chaulin ${ }^{1,2}$, Olga E. Abashina ${ }^{1}$, Dmitry V. Duplyakov ${ }^{1,2}$ \\ ${ }^{1}$ Samara State Medical University, Samara, Russia \\ 2 Samara Regional Clinical Cardiological Dispensary, Samara, Russia
}

Received 13 March 2020, Revised 26 May 2020, Accepted 7 August 2020

(C) 2020, Chaulin A.M., Abashina O.E., Duplyakov D.V.

(C) 2020, Russian Open Medical Journal

\begin{abstract}
Certain success has been achieved in the treatment of cancer due to the development of various effective chemotherapeutic drugs. However, an increase in their effectiveness (aggressiveness) was associated with a growth of undesirable effects on the entire human body, in particular, on the cardiovascular system. The damage to the cardiovascular system from chemotherapy in many cases is more significant than from the underlying disease. In recent years, a new direction of medicine has been formed - cardio-oncology. The major groups of cardiotoxic chemotherapeutic agents are anthracyclines, inhibitors of epidermal growth factor receptor type 2 (anti-HER2), antimetabolites, microtubule inhibitors, proteasome inhibitors, platinum-based chemotherapeutic drugs, and angiogenesis inhibitors (inhibitors of vascular endothelial growth factor). This review discusses principal pathophysiological mechanisms of the cardiotoxicity of these chemotherapeutic drugs.
\end{abstract}

Keywords: Cardiotoxicity, pathophysiology, anthracyclines, doxorubicin, epidermal growth factor receptor type 2 inhibitors, trastuzumab, antimetabolites, microtubule inhibitors, proteasome inhibitors, angiogenesis inhibitors.

Cite as Chaulin AM, Abashina OE, Duplyakov DV. Pathophysiological mechanisms of cardiotoxicity in chemotherapeutic agents. Russian Open Medical Journal 2020; 9: e0305.

Correspondence to Dmitry V. Duplyakov. E-mail: duplyakov@yahoo.com.

\section{Introduction}

Cardiotoxicity of medical preparations, as one of their side effects, has been known for a long time. The first description of cardiotoxic properties of pharmaceutical drugs in the literature dates back to 1967, when children, taking doxorubicin for leukemia, subsequently developed heart failure, which could lead to death even after successfully completing a course of chemotherapy. This finding prompted further study of the pathophysiological mechanisms underlying the cardiotoxic effect. Subsequent publications revealed some of those. High level of growth in morbidity and mortality from oncological diseases, as well as an increase in the resistance of a number of tumors to anticancer medications, created the need for the development and improvement of chemotherapeutic drugs, introduction of more aggressive treatment regimens, due to which it was possible to somewhat reduce the unfavorable trend. However, increasing the effectiveness of the anticancer treatment continues to go hand in hand with the progression of both short-term and long-term adverse cardiovascular effects [1]. This circumstance requires researchers to constantly study the pathophysiological mechanisms, understanding of which would improve diagnostic and cardioprotective measures [2].

Cardiotoxicity can be detected using functional and laboratory diagnostic methods, each of which has a number of advantages and disadvantages. Most of the main provisions (official guidelines) and clinical studies, conducted to determine cardiotoxicity, focus mainly on changes in functional diagnostics, and, specifically, on indicators of systolic myocardial function at rest, such as left ventricular ejection fraction (LVEF) and development of heart failure symptoms [2]. However, chemotherapy, besides a strong impact on LVEF at rest, has a very wide range of effects on the cardiovascular system, many of which occurs at the molecular and cellular levels and cannot be detected at early stages by functional diagnostic methods, due to their insufficient sensitivity. Although LVEF changes remain the keystone criterium for detecting cardiotoxicity of chemotherapy, there is a need to revise the definition and diagnostic criteria to include direct effects on the structure of the heart (for example, apoptosis and fibrosis), its diastolic function, cardiac conduction and arrhythmias, systemic hemodynamics, hemostatic processes, along with a heart reaction to trauma and stress [2]. At the same time, the pathophysiological mechanisms of these effects of chemotherapeutic agents are still poorly understood.

The main advantages of functional diagnostic methods, such as electrocardiography (ECG) and echocardiography (EchoCG), are wide availability and low cost. At the same time, low sensitivity is considered an apparent drawback of these functional diagnostic methods, which are characterized by late establishment of the fact of cardiotoxicity (irreversible damage and death of cardiomyocytes). Laboratory biomarkers of myocardial alteration, e.g., cardiac troponins T and I, as well as natriuretic peptides (BNP, NT-proBNP), are highly specific and more sensitive than conventional EchoCG and ECG parameters. With development of novel technologies on enhancing the detecting ability, troponin 
immunoassays have acquired the capability to determine lower troponin concentrations and thereby register even the smallest damage to the myocardium [3-5]. Thus, it has become possible to identify damage to cardiomyocytes at the earliest stages (at which these cells still retained high viability). This finding is certainly important, since early stages are reversible, that is, the timely use of cardioprotective measures would probably be able to reverse the development of pathophysiological changes.

Investigating cardiotoxicity of anticancer drugs is a relatively new, relevant field of study, as evidenced by the rapid growth in the numbers of publications over the past few years. However, existing review articles considered an issue of cardiotoxicity of anthracyclines (doxorubicin) and epidermal growth factor receptor type 2 inhibitors (trastuzumab) in sufficient detail, while insufficient attention was paid to other groups of anticancer pharmaceutical drugs [3]. In contrast to earlier publications, we have tried to comprehensively discuss the pathophysiological mechanisms underlying cardiotoxicity of major groups of anticancer medications, including those recently approved for clinical practice.

At present, the array of anticarcinogenic agents is very significant. Major classes (groups) and a list of chemotherapeutic preparations with significant cardiotoxicity are presented in Table 1. The chemical nature of cardiotoxic medications is very diverse and includes both high-molecular-weight protein molecules (monoclonal antibodies, for example, trastuzumab, pertuzumab, etc.) and low-molecular-weight compounds (smaller molecules, such as lapatinib, imatinib, and others) with variable pathophysiological effects.

It should also be noted that cardiotoxicity is inherent in a number of other compounds that are not anticarcinogenic, such as $\beta 2$-adrenomimetics (fenoterol, salbutamol, terbutaline), narcotic substances (cocaine, methamphetamine), etc. In addition, recent data indicate possible cardiotoxicity of statins, which, among other things, can increase against the background of physical activity and psychological and emotional overstrain [6]. This may be of clinical importance, since the combination of additional adverse effects on the myocardium is highly undesirable in such conditions. The simultaneous use of several pharmaceutical drugs with cardiotoxic effects should be avoided, and the adverse factors, contributing to cardiotoxicity should be eliminated, or at least reduced as much as possible.

Table 1. Major classes of cardiotoxic chemotherapeutic agents

\begin{tabular}{|c|c|}
\hline Class of pharmaceutical drugs & Most common medications \\
\hline Anthracyclines & $\begin{array}{c}\text { Doxorubicin, epirubicin, } \\
\text { daunorubicin, mitoxantrone }\end{array}$ \\
\hline $\begin{array}{c}\text { Epidermal growth factor receptor } \\
\text { type } 2 \text { inhibitors (anti-HER2 } \\
\text { medications) }\end{array}$ & $\begin{array}{l}\text { Trastuzumab, pertuzumab, ado- } \\
\text { trastuzumab emtansine, lapatinib, } \\
\text { imatinib }\end{array}$ \\
\hline Antimetabolites & $\begin{array}{l}\text { 5-Fluorouracil, capecitabine, } \\
\text { cytarabine, gemcitabine, } \\
\text { methotrexate, hydroxyurea }\end{array}$ \\
\hline Microtubule inhibitors & Paclitaxel, docetaxel \\
\hline Proteasome inhibitors & $\begin{array}{l}\text { Bortezomib, ixazomib, carlfilzomib, } \\
\text { marizonib, oprozomib, delanzomib }\end{array}$ \\
\hline $\begin{array}{l}\text { Platinum-based chemotherapeutic } \\
\text { drugs }\end{array}$ & Cisplatin, oxaliplatin \\
\hline $\begin{array}{l}\text { Angiogenesis inhibitors (vascular } \\
\text { endothelial growth factor inhibitors) }\end{array}$ & Bevacizumab, ramucirumab \\
\hline
\end{tabular}

The main efforts of scientists are aimed at studying the pathophysiological mechanisms of cardiotoxicity and searching for optimal methods of its detection and prevention at early stages. Establishment of molecular mechanisms would allow the development of cardioprotective medications to eliminate and reduce the harmful effects of chemotherapeutic drugs, while early detection of cardiotoxicity would permit to use cardioprotective measures at those early stages that may affect the further outcome. Since the discovery of cardiotoxicity as one of the types of side effects in chemotherapeutic drugs (1967) up to the present, a fairly large number of studies have been conducted to reveal the major pathophysiological mechanisms of cardiotoxicity. Our review is devoted to discussing the pathophysiological mechanisms of cardiotoxicity, inherent in each specific class of pharmaceutical drugs.

\section{Anthracyclines}

Anthracyclines are considered among the most effective anticancer pharmaceutical drugs currently in use. Anthracyclines are a class of antibiotics, produced by Streptomyces bacteria and discovered about 60 years ago. They are used to treat many different forms of cancer: lymphoma, leukemia, sarcoma, breast cancer, etc. [7]. The most commonly used anthracycline drugs in routine practice are doxorubicin, epirubicin, daunorubicin, and mitoxantrone [8].

Anthracyclines exhibit antitumor properties via four main mechanisms: 1) disruption of the nucleic acid synthesis (DNA and RNA) by intercalation between pairs of nitrogenous bases; 2) inhibition of topoisomerase II (which leads to DNA breaks and prevents its repair by ligation); 3) modification of histone proteins, weakening DNA recovery; 4 ) increase in formation of free radicals (reactive oxygen species), mediated by iron, which leads to damage to nucleic acids and intracellular protein-lipid structures $[7,9,10]$.

Although anthracyclines, due to their high antitumor efficacy, remain the most effective and widely used therapy, their use is limited by a number of factors. One of the limiting factors in the general use of anthracyclines is their ultimate cardiotoxicity. Heart failure and left ventricular (LV) dysfunction are the most dangerous short-term and long-term complications of anthracyclines, occurring in 5-23\% of patients, causing a decrease in their physical performance (i.e. disability) and an increase in mortality [11].

The risk of early and late cardiotoxicity of anthracyclines is highly dependent on the cumulative effects of anthracycline. At a cumulative dose of $400 \mathrm{mg} / \mathrm{m}^{2}$, there is a $5 \%$ risk of developing heart failure, which increases to $25 \%$ at $700 \mathrm{mg} / \mathrm{m}^{2}$ [12]. In patients with additional risk factors, such as age (under 18 and over 65), previous or underlying cardiovascular disease (hypertension, left ventricular hypertrophy, coronary artery disease, diabetes mellitus, prior or concomitant radiation therapy, to reduce the risk in the development of left ventricular dysfunction, it is recommended to decrease the total cumulative dose to $450 \mathrm{mg} / \mathrm{m}^{2}$. Even at low cumulative doses, e.g., such as the standard course of breast cancer therapy, approximately $20 \%$ of patients have a decrease in LV systolic function during the first 6 months of treatment [7]. The suppression of LV systolic function was explained by experimental studies on animals, demonstrating that anthracycline drugs cause atrophy of cardiomyocytes and a decrease in the LV mass. According to Ferreira de Souza T. et al. 
(2018), immediately after anthracycline therapy, the LV mass decreased, which indicated cardiomyocyte atrophy. Loss of LV mass was associated with the degree of damage to cardiomyocytes, assessed by peak troponin T levels [13]. Thus, every exposure to anthracycline, even minimal/recommended, still contained some risk of cardiac dysfunction. In such conditions, the exclusion of other adverse effects on the myocardium could become an important circumstance.

The mechanisms of anthracycline-induced cardiotoxicity studied to date are multiple and include the following pathogenetic links: oxidative stress, inhibition of topoisomerase II, inflammatory processes, and autophagy.

One of the earliest discovered and thoroughly studied mechanisms of alteration is the damaging effect of reactive oxygen species. It was found that anthracyclines cause multiple forms of cellular damage to cardiac myocytes and endothelial cells due to excess production of free radicals induced by the quinone group of these compounds [14]. Myocardial tissue is more susceptible to the damaging effect of free radicals, since cardiomyocytes are rich in mitochondria and have a relatively lower activity of the most important antioxidant defense enzymes (superoxide dismutase and catalase). In addition, another enzyme that protects cardiomyocytes from free radicals, glutathione peroxidase, is inhibited by doxorubicin. The accumulation of the toxic metabolite of doxorubicin, doxorubicinol, is an important link in the pathophysiological action of anthracyclines. Doxorubicin is about 50-500 times more potent than doxorubicin in reducing the systolic function of the heart; it also inhibits the calcium and sodium ion exchange pumps in mitochondria, sarcoplasmic reticulum and sarcolemma, which contributes to increased formation of free oxygen radicals [1].

Dexrazoxane, a derivative of ethylenediaminetetraacetate, chelates iron (i.e., forms complexes with iron ions), and as a result, reduces the number of metal ions that can bind to anthracycline, resulting in a decrease in the formation of iron-containing free radicals, which largely mediate the cardiotoxic effect of anthracyclines. Dexrazoxane was formulated to provide cardiac protection against anthracycline cardiotoxicity by reducing the formation of superoxide radicals. Dexrazoxane has been found to provide cardiac protection against anthracyclines in women with advanced breast cancer [15]. Also, some data indicate an alternative mechanism, in which the binding of dexrazoxane to topoisomerase $2 \beta$ leads to the degradation of this enzyme [16]. However, dexrazoxane has not gained widespread use in the prevention of anthracycline cardiotoxicity, in part because of concerns about a decrease in the antitumor activity (effects) of anthracyclines, a role in the development of secondary malignancy, and also because of its teratogenic and embryotoxic properties. Perhaps a new understanding of the effect of dexrazoxane on topoisomerase $2 \beta$, as a mechanism for the prevention of anthracycline-induced cardiotoxicity, would lead to revision of its clinical utility.

Among the pathophysiological mechanisms of anthracycline cardiotoxicity, an important role is played by the effect of anthracyclines on topoisomerase II. Recently, the attention of researchers has been drawn to the contribution to anthracyclineinduced cardiotoxicity rather than solely to the role of topoisomerase II as a molecular target responsible for the antitumor properties of anthracyclines. Topoisomerases are essential enzymes required for DNA transcription, replication and/or recombination. In humans, two topoisomerase II isoenzymes are expressed: topoisomerase Ila and topoisomerase $\| \beta$. Significant expression of topoisomerase Ila is noted in rapidly proliferating cells, while topoisomerase $\| \beta$ is expressed in slowly dividing or non-dividing cells, such as cardiomyocytes in the postnatal period [17]. Mice, lacking topoisomerase $\| \beta$, are protected from DNA damage, caused by anthracycline and cardiomyocyte death [8]. A recent genome-wide association study identified the retinoic acid receptor gamma (RAR- $\gamma$ ) as a susceptibility gene for anthracycline-induced cardiotoxicity, where genetic variants of RAR- $y$ alter the effects of retinoic acid on topoisomerase $\| \beta$ expression in vitro. These results have led to several new avenues of research regarding, whether changes in topoisomerase $\| \beta$ could provide greater cardiac protection in the course of anthracycline treatment [18]. In theory, if an anthracycline chemotherapy is designed to selectively target topoisomerase Ila, but not topoisomerase $\| \beta$, then the cardiotoxic effects of such anthracycline preparations could be weakened. In addition, by studying/determining the expression of topoisomerase $\| \beta$, the risk of anthracycline-induced cardiotoxicity can be better predicted/prognosticated. Hence, identifying the pathophysiological mechanisms of the effect of anthracyclines on topoisomerase II opened a novel direction of research for the development of cardiac protection.

There is some evidence that inflammatory processes play a role in the pathogenesis of doxorubicin-induced cardiotoxicity. Doxorubicin causes the release of inflammatory mediators, such as histamine, tumor necrosis factor alpha and pro-inflammatory interleukins from monocytes and macrophages. These cytokines have functional receptors in the myocardium, which can lead to the development of dilated cardiomyopathy. Jadapalli J.K. et al. (2018), in their experimental study of mice, found that doxorubicin causes spleen contraction and irreversible dysregulation of cyclooxygenase and lectin-like oxidized low-density lipoprotein receptor-1, which was accompanied by increased myocardial inflammation [19].

It has also been reported that autophagy may play an important role in the pathogenesis of doxorubicin cardiotoxicity. However, due to the inconsistency of studies, investigating the effect of doxorubicin on cardiac autophagy and its significance for cardiotoxicity, more research is needed [20].

Very pronounced arrhythmogenic effect of doxorubicin is another important issue. It has been proven that doxorubicin affects the action potential of cardiomyocytes, channels, and the dynamics of intracellular calcium concentration [21-23]. Microscopic changes that may be responsible for the arrhythmogenic effects of doxorubicin include: destruction of the sarcolemma of cardiomyocytes in the region of the intercalated disc, myofibrillary disorder, dissociation of cardiomyocytes in muscle fibers, potentially leading to a slowdown in speed and conduction disturbances, and a decrease in the expression and function of connexin 43. The latter is the main molecular component of gap junctions, which are responsible for rapid electrical synchronization throughout the myocardium [23, 24].

Another important factor contributing to anthracyclineinduced cardiotoxicity is the function of membrane transport systems, which modulate the concentration of anthracyclines inside cells, including cardiomyocytes, and, as a consequence, the strength of the effect of these medications. Efflux, i.e. the outflow (release) of anthracyclines from the cell, is partially regulated by 
the expression of membrane transporters of the adenosine triphosphate-binding cassette $(A B C)$ family, including proteins with multidrug resistance (MDR), also known as P-glycoprotein. The role of MDR proteins in the development of cardiotoxicity in humans has been demonstrated by candidate gene studies, as well as genome-wide studies that have identified an interaction between MDR1 polymorphisms and cardiotoxicity $[25,26]$. A recent study confirmed that breast cancer patients, who developed cardiotoxicity, had half the expression levels of the $A B C B 1$ messenger RNA (adenosine triphosphate-binding cassette B1), encoding the MDR1 protein. This finding has a direct clinical importance in cancer patients receiving other drugs that are substrates of MDR, such as calcium channel blockers (e.g., verapamil, diltiazem), which could raise the intracellular level of cardiotoxic chemotherapeutic drugs, potentially increasing the risk of cardiotoxicity [25].

Besides, anthracycline has recently been shown to decrease the number of cardiac mesenchymal progenitor cells and the circulating progenitor cell population, and, accordingly, reduce the heart's ability to cardiac repair, especially when it is stressed [27, 28]. In children, the number of mesenchymal progenitor cells is higher than in adults, which may explain the delayed development of heart failure in them in response to anthracycline therapy.

Thus, despite the fact that anthracyclines have been an effective therapeutic agent for over 50 years, further study of molecular pathways is necessary to develop innovative strategies to treat and mitigate anthracycline-induced toxicity. Highly sensitive methods for measuring troponins have shown that the myocardium is a very sensitive tissue to many adverse effects (stress, exercise, statins, etc.) [3-5]. A combination of these factors with cardiotoxic properties of anthracyclines is highly undesirable. Therefore, among the measures to mitigate cardiotoxicity, taking into account the data discussed above, we can recommend the following: reducing stress and physical exertion, reducing the dose of statin or switching to alternative lipid-lowering drugs, reducing the intake of calcium channel blockers, as well as using the cardioprotective medicine dexrazoxane.

\section{Anti-HER2 drugs}

HER2-targeted agents are a class of pharmaceutical drugs that specifically inhibit human epidermal growth factor receptors 2 (also known as ErbB2), which are members of the epidermal growth factor family of receptor tyrosine kinases. Prior to the development of HER2-targeted therapy, HER2-positive (HER2+) breast cancer patients, characterized by overexpression of HER2 receptors, had a poor prognosis due to the aggressive nature and rapid tumor growth. Trastuzumab was the first HER2-targeted chemotherapeutic drug approved by the US Food and Drug Administration in 1998 for the treatment of advanced metastatic cancer, and then its indications were expanded in 2007 to treat early stage HER2+ breast cancer $[29,30]$. Chemically, trastuzumab is a humanized monoclonal antibody that specifically targets the HER2 receptor (ErbB2), located on the cell membrane of an atypical cell. Inhibition of HER2 receptors impairs phosphorylation (activation) of intracellular tyrosine kinases, which are critical regulators of cell growth and survival. Clinical studies have shown that trastuzumab improves overall survival in advanced (metastatic) breast cancer from 20 to 25 months [31]. For early stages of breast cancer, trastuzumab therapy reduces the absolute risk of cancer recurrence after surgery by $9.5 \%$, and the absolute mortality risk by $3 \%$. Exposure to trastuzumab, besides positive antitumor effects, led to some negative consequences in the form of minor cardiac dysfunction. The absolute risk of decreased LV function was $2.1 \%$, and it usually declined after discontinuation of the therapy [32].

HER2-targeted agents can cause asymptomatic cardiac dysfunction in some patients and, less commonly, overt congestive heart failure. Although the clinical significance of asymptomatic LV dysfunction caused by trastuzumab remains unknown, clinicians should take this fact into account. The cardiac dysfunction Caused by HER2 chemotherapy is exOlained by impaired signaling between the HER2 receptor and its ligand, a growth factor called neuregulin. The ligand-receptor (neuregulin-HER2) signaling pathway is critical for the normal growth of cardiomyocytes, as well as for survival and maintenance of homeostasis [33]. Thus, presumably, HER2-targeted agents can cause a decrease in ejection fraction due to the effect on myocytic neuregulin-HER2 signaling, required for normal cardiac recovery and myocyte homeostasis.

In addition, neuregulin-HER2 signaling regulates other functions in the cardiovascular system, including vasomotor tone and the sympathetic nervous system. Breast cancer patients treated with an anti-HER2 medication have elevated norepinephrine levels along with increased blood pressure and heart rate [34]. In preclinical models, the expression and activity of $\beta$-adrenergic receptors is associated with the expression of HER2, while HER2 activation is a critical modulator of the toxicity of chronic exposure to $\beta$-adrenoceptor agonists. Consequently, an additional mechanism of HER2-targeted therapy that alters cardiac function may be associated with a chronic increase in sympathetic tone $[35,36]$. Further research is needed to evaluate the effect of HER2-targeted therapy on sympathetic tone, and to investigate the potential role of $\beta$-blockers in alleviating cardiac dysfunction associated with HER2 inhibitors.

Histological changes after exposure to trastuzumab differ from those after the use of anthracyclines. Researchers from Michigan State University (USA) studied early histopathological changes in the myocardium of rabbits after subcutaneous administration of trastuzumab. Rabbit myocardium was largely infiltrated with lymphocytes and macrophages. Transmission microscopy showed edema with separation of myofibril bundles and rupture of sarcomeres. Changes in the mitochondria of cardiomyocytes included slight disorganization of the cristae. Rabbits treated with sodium selenite prior to trastuzumab administration had less pronounced changes, which may be indicative of the preventive cardioprotective properties of selenium [37].

In the past few years, new low-molecular-weight HER2targeted agents have emerged. Among these, lapatinib is an oral HER2-targeted small molecule that inhibits both the HER2/neu receptor and the HER2/neu receptor tyrosine kinase, which, in its turn, triggers an intracellular cascade of responses.

Pertuzumab is a recombinant monoclonal antibody against HER2 designed to overcome resistance to trastuzumab [30, 38]. Ado-trastuzumab emtansine is one of the newest anti-HER2 drugs approved by the US Food and Drug Administration for the treatment of metastatic breast cancer that is resistant to trastuzumab [39]. Ado-trastuzumab emtansine acts as an antibody-drug conjugate with a monoclonal antibody to the HER2 receptor. This antibody-drug conjugate provides a preferential 
intracellular delivery of a chemotherapeutic drug mainly to HER2+ tumor cells, thereby reducing toxic effects on other tissues and cells, in particular on cardiovascular system, while maintaining a high antitumor efficacy.

At present, routine monitoring of cardiac function during treatment with HER2-targeted therapy is considered necessary. It is recommended to assess LVEF every 3 months during HER2targeted therapy and to start treatment for heart failure if LVEF falls below $40 \%$. Consideration should also be given to this therapy if a patient has a drop in LVEF $>10$ points below baseline with an initial LVEF $\geq 50 \%$ [40].

HER-targeted therapy has significantly improved outcomes in breast cancer patients, and its applicability is expanding for the treatment of advanced gastric cancer that overexpresses HER2+ receptors [41]. With continued use of HER-targeted therapy, potential cardiovascular side effects are expected due to the coincidence of biological effects, mediated by the activation of HER receptors (ErbB) among the cells of various tumors and cardiovascular system.

\section{Antimetabolites}

Antimetabolites are a class of medicines that interfere with the biosynthesis of nucleic acids by replacing normal DNA/RNA nucleotides and thereby damage proliferating cells during the synthetic phase ( $\mathrm{S}$ phase) of mitosis. The most common and wellknown antimetabolites include 5-fluorouracil, capecitabine, cytarabine, gemcitabine, methotrexate, and hydroxyurea, which are commonly used to treat leukemia, as well as ovarian, breast, gastrointestinal, and other solid tumors. 5-Fluorouracil is the most widely used antimetabolite with cardiotoxicity rates ranging from $1 \%$ to $7.6 \%$ [42]. The most commonly reported cardiac effects, associated with 5-fluorouracil, are myocardial ischemia, angina pectoris, chest pain, and ECG changes (ST segment changes and T wave abnormalities). The incidence of ischemia associated with 5fluorouracil is higher in patients with pre-existing coronary artery disease $(4.5 \%)$ compared with patients who did not have it (1.1\%). Reuse of 5-fluorouracil usually reproduces the ischemic syndrome and symptoms. Cardiotoxicity usually occurs early (during the first to third dose) and is more common after higher doses and continuous infusions of the medication [43]. The effectiveness of preventive measures is currently controversial. Thus, in standard guidelines, prophylactic regimens with antiplatelet agents, nitroglycerin, or calcium channel blockers are not recommended due to lack of their effectiveness. However, there are two smaller studies, in which the prophylactic use of calcium channel blockers or nitrates under the conditions of anticancer treatment with 5fluorouracil or capecitabine resulted in improvement of anginal symptoms [44, 45].

The pathophysiology of the cardiotoxicity of 5-fluorouracil and capecitabine appears to be multifactorial. Recent studies have shown that the toxicity of antimetabolites leads to endothelial damage with subsequent development of thrombosis; increased metabolism, resulting in depletion of energy and development of ischemia; oxidative stress, causing cell damage; spasm of the coronary arteries, leading to myocardial ischemia; as well as a decrease in the ability of erythrocytes to carry and give oxygen to tissues (due to an increase in the oxygen affinity of hemoglobin), causing myocardial ischemia [46].

\section{Microtubule inhibitors}

Microtubule inhibitors, also known as taxanes (alkaloids of Pacific yew, Taxus brevifolia Nutt.), include two major preparations: paclitaxel and docetaxel. Taxanes have antitumor effects by disrupting microtubule function. Microtubules are protein structures that are part of the cytoskeleton and are necessary for cell division, while taxanes stabilize guanosine diphosphate-bound tubulin in microtubules, thereby inhibiting the process of cell division (mitosis). Taxanes are used in the treatment of many solid tumors, including breast and ovarian cancers $[47,48]$. After the introduction of paclitaxel into clinical practice, its cardiotoxic effects were immediately discovered, especially the development of cardiac arrhythmias. Bradycardia was common, as well as heart block (atrioventricular, bundle branch), on the basis of which it was suggested that microtubules play a role in the regulation of intracellular calcium levels. The researchers found that paclitaxel exposure significantly affected the dynamics of intracellular calcium levels in different phases of cardiac activity. In the experimental model on isolated cardiomyocytes, a decrease in time from the state of maximum contraction to relaxation was noted, which predisposed heart to various arrhythmias [49].

As a rule, bradyarrhythmias are asymptomatic, they quickly stop by themselves after the end of the taxane-taking course. Supraventricular arrhythmias, including atrial fibrillation, atrial flutter, and atrial tachycardia, also occur as side-effects of taking taxanes. Although their pathogenesis can be multifactorial, one of the provoking factors might be polyethoxylated castor oil - the paclitaxel delivery vehicle, which induces histamine release [50]. Data from a number of clinical studies imply that paclitaxel is capable of contributing to the development of LV dysfunction [51]. A preclinical study examining the effects of paclitaxel per se, as well as in combination with trastuzumab, showed that simultaneous inhibition of the HER2 (ErbB2) receptors and treatment with paclitaxel had an additive deteriorating effect on adult cardiomyocytes, manifested mainly in myofibrillar structure and function changes, but not causing death of myocytes [52]. Cardiac dysfunction developed in $20 \%$ of patients who received paclitaxel and doxorubicin simultaneously, against the background of much lower cumulative doses than with doxorubicin alone [53]. Hence, the combination of trastuzumab and doxorubicin with taxanes may be accompanied by a higher incidence of cardiac dysfunction, emphasizing the need for further prospective studies, investigating the cardiovascular effects of several chemotherapeutic drugs used simultaneously, in order to clarify synergistic and additive effects on the cardiovascular system.

\section{Proteasome inhibitors}

The ubiquitin-proteasome system regulates protein metabolism and activation of intracellular signaling pathways, including tumor growth, and promotes cell survival by regulating apoptosis. Chemotherapeutic drugs targeting proteasomes have found widespread use and have shown high efficiency in the treatment of oncological diseases. However, these pharmaceutical drugs are also associated with the development of heart failure, since the proteasome plays a fundamental role in maintaining the structure of heart cells and, accordingly, their functioning [54]. Bortezomib was the first proteasome inhibitor used in clinical practice to suppress the proliferation of plasma cells in multiple myeloma. There are relatively few reports of the onset of heart failure in the course of treatment with bortezomib $[55,56]$. Due to 
relatively low incidence of heart failure, many researchers consider it relatively safe. However, new proteasome inhibitors are currently being developed, including carfilzomib, marizonib, oprozomib, irreversible and more aggressive proteasome inhibitors. Carfilzomib is currently approved as a second line treatment for recurrent multiple myeloma. Although this therapy appears to be more effective in cases of resistant myeloma than previous chemotherapeutic drugs, there may be a greater likelihood of cardiovascular events, including heart failure, sudden cardiac death, and acute coronary syndrome [57]. Further studies will clarify the cardiovascular safety of carfilzomib and other proteasome inhibitors.

\section{Platinum-based chemotherapeutic drugs}

One of the most undesirable effects of platinum-based chemotherapeutic drugs, used to treat many solid tumors (genitourinary, gastrointestinal, lung, head and neck tumors), is vascular toxicity. A common and effective platinum-based chemotherapeutic drug is cisplatin, with a cure rate approaching $90 \%$ in patients with testicular cancer. However, with such high cure rate value, there is a very high risk of cardiovascular complications $[58,59]$.

Platinum-based antineoplastic medications induce intrastrand crosslinking of DNA, thereby interfering with vital cellular processes such as replication and transcription. Ultimately, these crosslinks inhibit DNA repair and synthesis in cancer cells, leading to cellular apoptosis. Platinum-based anticancer agents are sometimes referred to as alkylating agents by their mechanism of action, but they do not have an alkyl group in their composition [60].

The most studied consequences of late vascular toxicity of chemotherapy with platinum-based drugs include hypertension, dyslipidemia, early atherosclerosis, ischemic heart disease, and Raynaud's phenomenon [61, 62]. Cancer survivors have an increased risk of developing both adverse cardiovascular risk factors (obesity, hypertension, hyperlipidemia, diabetes mellitus) and cardiovascular diseases [62].

Elimination of cisplatin from the body is extremely slow: platinum concentrations have been measured in blood serum over several years. It is assumed that circulating platinum, continuously acting on the endothelium, leads to its damage and dysfunction, which adversely affects the cardiovascular system. Research shows that endothelial dysfunction develops in patients treated with cisplatin for testicular cancer. This is supported by the data, according to which, in patients taking cisplatin, the levels of endothelial damage biomarkers, such as von Willebrand factor, tissue plasminogen activator and type 1 plasminogen activator inhibitor, were significantly higher than in the control group. Coronary artery disease often develops in patients who survived platinum-based chemotherapy. In addition, cisplatin treatment induces thickening of the intima-media in the carotid artery wall [63]. Taking into account these mechanisms of action and observation, it can be assumed that platinum-based chemotherapeutic drugs increase the risk of atherosclerosis, which needs further confirmation. Unlike anthracyclines, platinum-based anticancer agents do not induce systolic dysfunction [64].

Angiogenesis inhibitors (vascular endothelial growth factor inhibitors)
Vascular endothelial growth factors (VEGFs) are important for angiogenesis, and participate in tumor growth as well. Vital processes, regulated by VEGF, include angiogenesis, endothelial cell survival (via blocking their apoptosis), blood pressure control by means of vasodilation, and influence on the contractile function of the heart [65]. Given such physiological role of VEGF, serious effects of VEGF-inhibiting anticancer medications are somewhat expected.

Arterial hypertension is among the most common adverse effects reported for VEGF inhibitors. The hypertensive effect of VEGF blockers is dose related, as hypertension is more common in individuals receiving higher dosages [66]. In the pathogenesis of hypertension caused by VEGF inhibitors, the following pathogenetic links are distinguished: an increase in the total peripheral vascular resistance, a weakening of vasorelaxation, and a dysfunction of the renal glomeruli. For example, VEGFs activate endothelial nitric oxide synthase (eNOS), due to which vasorelaxation occurs, while VEGF inhibitors, on the contrary, suppress this mechanism of vascular relaxation $[67,68]$. In mice with VEGF deficiency, pathological changes in the renal glomeruli were noted in the form of damage to the basement membrane and loss of podocytes, which are important elements of the renal filter. This mechanism explains the proteinuria observed in patients taking VEGF inhibitors $[69,70]$.

Besides, the use of VEGF inhibitors is associated with an increased risk of arterial thromboembolic complications. According to F.A. Scappaticci et al., in patients taking bevacizumab (monoclonal antibody against VEGF), the risk of arterial thromboembolic events (stroke, transient ischemic attacks, myocardial infarction) undergoes a threefold increase. This can be explained by the fact that activation of platelets results in the release of VEGF, which regulates the survival and regeneration of endothelial cells, along with a restoration of vascular microcirculation, thereby providing antiplatelet role [71]. In conditions of VEGF inhibition by anticancer medications, the balance between these pro-thrombotic and antiplatelet mechanisms is violated, which leads to thrombosis.

In some patients, receiving VEGF inhibitors, the development of hypertrophy and systolic dysfunction was observed. Apparently, VEGFs, regulating the growth of the myocardial microvasculature, provide adaptation of the heart to arterial pressure overload [72]. However, in conditions of VEGF deficiency, the development of the microvasculature lags behind the growth of cardiomyocytes, which leads to cardiac remodeling and more rapid development of heart failure decompensation.

\section{Conclusion}

The presented review shows that cardiotoxicity is a very common adverse consequence of using nearly all major currently existing anti-cancer drugs: anthracyclines, inhibitors of the epidermal growth factor receptor type 2 (anti-HER2), antimetabolites, microtubule inhibitors, proteasome inhibitors, platinum-based chemotherapeutic drugs, inhibitors of angiogenesis (inhibitors of vascular endothelial growth factor). The pathophysiological mechanisms underlying the undesirable effects on the cardiovascular system are very abundant and include: increased oxidative stress, autophagy, apoptosis and inflammatory processes, the effect on hemostasis and systemic hemodynamics, as well as suppression of cardiac contractile function. A detailed 
study of the cardiotoxicity mechanisms of each specific drug would help developing necessary cardioprotective measures.

\section{Conflict of interest}

Authors declare no conflict of interest.

\section{Funding}

This study was not funded.

\section{References}

1. Pai VB, Nahata MC. Cardiotoxicity of chemotherapeutic agents: incidence, treatment and prevention. Drug Saf 2000; 22(4): 263-302. https://doi.org/10.2165/00002018-200022040-00002.

2. Lenneman CG, Sawyer DB. Cardio-Oncology: An Update on Cardiotoxicity of Cancer-Related Treatment. Circ Res 2016; 118(6): 1008-1020. https://doi.org/10.1161/circresaha.115.303633.

3. Sawaya H, Sebag IA, Plana JC, Januzzi JL, Ky B, Cohen V, et al. Early detection and prediction of cardiotoxicity in chemotherapy-treated patients. Am J Cardiol 2011; 107(9): 1375-1380. https://doi.org/10.1016/j.amjcard.2011.01.006

4. Jones M, O'Gorman P, Kelly C, Mahon N, Fitzgibbon MC. High-sensitive cardiac troponin-I facilitates timely detection of subclinical anthracycline-mediated cardiac injury. Ann Clin Biochem 2017; 54(1): 149-157. https://doi.org/10.1177/0004563216650464.

5. Chaulin AM, Karslyan LS, Bazyuk EV, Nurbaltaeva DA, Duplyakov DV. Clinical and Diagnostic Value of Cardiac Markers in Human Biological Fluids. Kardiologiia 2019; 59(11): 66-75. Russian. https://doi.org/10.18087/cardio.2019.11.n414.

6. Ünlü S, Nurkoç SG, Sezenöz B, Cingirt M, Gülbahar Ö, Abacı A. Impact of statin use on high sensitive troponin $\mathrm{T}$ levels with moderate exercise. Acta Cardiol 2019; 74(5): 380-385. https://doi.org/10.1080/00015385.2018.1510801.

7. Geisberg CA, Sawyer DB. Mechanisms of anthracycline cardiotoxicity and strategies to decrease cardiac damage. Curr Hypertens Rep 2010; 12(6): 404-410. https://doi.org/10.1007/s11906-010-0146-y.

8. Vasanthakumar A, Kattusamy K, Prasad R. Regulation of daunorubicin biosynthesis in Streptomyces peucetius - feed forward and feedback transcriptional control. J Basic Microbiol 2013; 53(8): 636-644. https://doi.org/10.1002/jobm.201200302.

9. Zhang S, Liu X, Bawa-Khalfe T, Lu LS, Lyu YL, Liu LF, et al. Identification of the molecular basis of doxorubicin-induced cardiotoxicity. Nat Med 2012; 18(11): 1639-1642. https://doi.org/10.1038/nm.2919.

10. Pang B, Qiao X, Janssen L, Velds A, Groothuis T, Kerkhoven R, et al. Drug-induced histone eviction from open chromatin contributes to the chemotherapeutic effects of doxorubicin. Nat Commun 2013; 4: 1908. https://doi.org/10.1038/ncomms2921.

11. Cardinale D, Colombo A, Lamantia G, Colombo N, Civelli M, De Giacomi $\mathrm{G}$, et al. Anthracycline-induced cardiomyopathy: clinical relevance and response to pharmacologic therapy. J Am Coll Cardiol 2010; 55(3): 213220. https://doi.org/10.1016/j.jacc.2009.03.095.

12. Shan K, Lincoff AM, Young JB. Anthracycline-induced cardiotoxicity. Ann Intern Med 1996; 125(1): 47-58. https://doi.org/10.7326/00034819-125-1-199607010-00008

13. Ferreira de Souza T, Quinaglia A C Silva T, Osorio Costa F, Shah R, Neilan TG, Velloso L, et al. Anthracycline Therapy Is Associated With Cardiomyocyte Atrophy and Preclinical Manifestations of Heart Disease. JACC Cardiovasc Imaging 2018; 11(8): 1045-1055. https://doi.org/10.1016/j.jcmg.2018.05.012.

14. Horenstein MS, Vander Heide RS, L'Ecuyer TJ. Molecular basis of anthracycline-induced cardiotoxicity and its prevention. Mol Genet Metab 2000; 71(1-2): 436-444. https://doi.org/10.1006/mgme.2000.3043.
15. Swain SM, Whaley FS, Gerber MC, Weisberg S, York M, Spicer D, et al. Cardioprotection with Dexrazoxane for doxorubicin-containing therapy in advanced breast cancer. J Clin Oncol 1997; 15(4): 1318-1332. https://doi.org/10.1200/jco.1997.15.4.1318.

16. Vejpongsa $\mathrm{P}$, Yeh ET. Topoisomerase $2 \beta$ : a promising molecular target for primary prevention of anthracycline-induced cardiotoxicity. Clin Pharmacol Ther 2014; 45-52. https://doi.org/10.1038/clpt.2013.201.

17. Lyu YL, Kerrigan JE, Lin CP, Azarova AM, Tsai $Y C$, Ban $Y$, et al. Topoisomerase Ilbeta mediated DNA double-strand breaks: implications in doxorubicin cardiotoxicity and prevention by dexrazoxane. Cancer Res 2007; 67(18): 8839-8846. https://doi.org/10.1158/0008-5472.can-07-1649.

18. Aminkeng $F$, Bhavsar AP, Visscher $H$, Rassekh SR, Li Y, Lee JW, et al. A coding variant in RARG confers susceptibility to anthracycline-induced cardiotoxicity in childhood cancer. Nat Genet 2015; 47(9): 1079-1084. https://doi.org/10.1038/ng.3374

19. Jadapalli JK, Wright GW, Kain V, Sherwani MA, Sonkar R, Yusuf N, et al. Doxorubicin triggers splenic contraction and irreversible dysregulation of COX and LOX that alters the inflammation-resolution program in the myocardium. Am J Physiol Heart Circ Physiol 2018; 315(5): H1091H1100. https://doi.org/10.1152/ajpheart.00290.2018.

20. Dirks-Naylor AJ. The role of autophagy in doxorubicin-induced cardiotoxicity. Life Sci 2013; 93(24): 913-916. https://doi.org/10.1016/i.Ifs.2013.10.013.

21. Milberg P, Fleischer D, Stypmann J, Osada N, Mönnig G, Engelen MA, et al. Reduced repolarization reserve due to anthracycline therapy facilitates torsade de pointes induced by IKr blockers. Basic Res Cardiol 2007; 102(1): 42-51. https://doi.org/10.1007/s00395-006-0609-0.

22. Wu Y, Anderson ME. CaMKII in sinoatrial node physiology and dysfunction. Front Pharmacol 2014; 5: 48 https://doi.org/10.3389/fphar.2014.00048.

23. Jensen RA, Acton EM, Peters JH. Doxorubicin cardiotoxicity in the rat: comparison of electrocardiogram, transmembrane potential, and structural effects. J Cardiovasc Pharmacol 1984; 6(1): 186-200. https://pubmed.ncbi.nlm.nih.gov/6199603.

24. Pecoraro $M$, Rodríguez-Sinovas $A$, Marzocco $S$, Ciccarelli $M$, laccarino G, Pinto A, et al. Cardiotoxic Effects of Short-Term Doxorubicin Administration: Involvement of Connexin 43 in Calcium Impairment. Int J Mol Sci 2017; 18(10): 2121. https://doi.org/10.3390/ijms18102121.

25. McCaffrey TA, Tziros C, Lewis J, Katz R, Siegel R, Weglicki W, et al. Genomic profiling reveals the potential role of TCL1A and MDR1 deficiency in chemotherapy-induced cardiotoxicity. Int J Biol Sci 2013 ; 9(4): 350-360. https://doi.org/10.7150/ijbs.6058.

26. Deng S, Wojnowski L. Genotyping the risk of anthracycline-induced cardiotoxicity. Cardiovasc Toxicol 2007; 7(2): 129-134. https://doi.org/10.1007/s12012-007-0024-2.

27. De Angelis A, Piegari E, Cappetta D, Marino L, Filippelli A, Berrino L, et al. Anthracycline cardiomyopathy is mediated by depletion of the cardiac stem cell pool and is rescued by restoration of progenitor cell function. Circulation 2010; 121(2): 276-292. https://doi.org/10.1161/circulationaha.109.895771.

28. Huang C, Zhang X, Ramil JM, Rikka S, Kim L, Lee $Y$, et al. Juvenile exposure to anthracyclines impairs cardiac progenitor cell function and vascularization resulting in greater susceptibility to stress-induced myocardial injury in adult mice. Circulation 2010; 121: 675-683. https://doi.org/10.1161/circulationaha.109.902221.

29. FDA expands use of herceptin for early-stage breast cancer. Mayo Clin Womens Healthsource 2007; 11(6): 3. https://pubmed.ncbi.nlm.nih.gov/17476181.

30. Artamonova EV, Manzyuk LV. HER2-positive metastatic breast cancer: new therapeutic options. Tumors of Female Reproductive System 2013; (3-4): 60-66. Russian. https://doi.org/10.17650/1994-40982013-0-3-4-60-66. 
31. Slamon DJ, Leyland-Jones B, Shak S, Fuchs H, Paton V, Bajamonde A, et al. Use of chemotherapy plus a monoclonal antibody against HER2 for metastatic breast cancer that overexpresses HER2. N Engl J Med 2001; 344(11): 783-792. https://doi.org/10.1056/nejm200103153441101.

32. Moja L, Tagliabue L, Balduzzi S, Parmelli E, Pistotti V, Guarneri V, et al. Trastuzumab containing regimens for early breast cancer. Cochrane Database Syst Rev 2012; 2012(4): CD006243. https://doi.org/10.1002/14651858.cd006243.pub2.

33. Pentassuglia L, Sawyer DB. The role of Neuregulin-1beta/ErbB signaling in the heart. Exp Cell Res 2009; 315(4): 627-637. https://doi.org/10.1016/j.yexcr.2008.08.015.

34. Lenneman CG, Abdallah WM, Smith HM, Abramson V, Mayer IA, Silverstein C, et al. Sympathetic nervous system alterations with HER2+ antagonism: an early marker of cardiac dysfunction with breast cancer treatment? Ecancermedicalscience 2014; 8: 446. https://doi.org/10.3332/ecancer.2014.446.

35. Kuramochi Y, Lim CC, Guo X, Colucci WS, Liao R, Sawyer DB. Myocyte contractile activity modulates norepinephrine cytotoxicity and survival effects of neuregulin-1beta. Am J Physiol Cell Physiol 2004; 286(2): C222-C229. https://doi.org/10.1152/ajpcell.00312.2003.

36. Sysa-Shah P, Tocchetti CG, Gupta M, Rainer PP, Shen X, Kang BH, et al. Bidirectional cross-regulation between erbb2 and beta-adrenergic signalling pathways. Cardiovasc Res 2015; 109(3): 358-373. https://doi.org/10.1093/cvr/cvv274.

37. Laird-Fick H.S., Tokala H., Kandola S., Kehdi M., Pelosi A., Wang L., et al. Early morphological changes in cardiac mitochondria after subcutaneous administration of trastuzumab in rabbits: possible prevention with oral selenium supplementation. Cardiovasc Pathol 2019. 44: 107159. https://doi.org/10.1016/j.carpath.2019.107159.

38. Singh JC, Jhaveri K, Esteva FJ. HER2-positive advanced breast cancer: optimizing patient outcomes and opportunities for drug development. $\mathrm{Br} J$ Cancer 2014; 111(10): 1888-1898. https://doi.org/10.1038/bjc.2014.388.

39. Verma S, Miles D, Gianni L, Krop IE, Welslau M, Baselga J, et al; EMILIA Study Group. Trastuzumab emtansine for HER2-positive advanced breast cancer. N Engl J Med 2012; 367(19): 1783-1791. https://doi.org/10.1056/nejmoa1209124.

40. Mackey JR, Clemons M, Côté MA, Delgado D, Dent S, Paterson A, et al. Cardiac management during adjuvant trastuzumab therapy: recommendations of the Canadian Trastuzumab Working Group. Curr Oncol 2008; 15(1): 24-35. https://doi.org/10.3747/co.2008.199.

41. Bang YJ, Van Cutsem E, Feyereislova A, Chung HC, Shen L, Sawaki A, et al; ToGA Trial Investigators. Trastuzumab in combination with chemotherapy versus chemotherapy alone for treatment of HER2positive advanced gastric or gastro-oesophageal junction cancer (ToGA): a phase 3, open-label, randomised controlled trial. Lancet 2010; 376(9742): 687-697. https://doi.org/10.1016/s01406736(10)61121-x.

42. Alter P, Herzum M, Soufi M, Schaefer JR, Maisch B. Cardiotoxicity of 5fluorouracil. Cardiovasc Hematol Agents Med Chem 2006; 4(1): 1-5. https://doi.org/10.2174/187152506775268785.

43. de Forni $M$, Malet-Martino MC, Jaillais $P$, Shubinski RE, Bachaud JM, Lemaire $L$, et al. Cardiotoxicity of high-dose continuous infusion fluorouracil: a prospective clinical study. J Clin Oncol 1992; 10(11): 1795-1801. https://doi.org/10.1200/jco.1992.10.11.1795

44. Cianci G, Morelli MF, Cannita K, Morese R, Ricevuto E, Di Rocco ZC, et al. Prophylactic options in patients with 5-fluorouracil-associated cardiotoxicity. $\mathrm{Br} J$ Cancer 2003; 88(10): 1507-1509. https://doi.org/10.1038/sj.bjc.6600967.

45. Ambrosy AP, Kunz PL, Fisher GA, Witteles RM. Capecitabine-induced chest pain relieved by diltiazem. Am J Cardiol 2012; 110(11): 16231626. https://doi.org/10.1016/j.amjcard.2012.07.026.

46. Polk A, Vistisen K, Vaage-Nilsen M, Nielsen DL. A systematic review of the pathophysiology of 5-fluorouracil-induced cardiotoxicity. BMC
Pharmacol Toxicol 2014; 15: 47. https://doi.org/10.1186/2050-651115-47.

47. Abal M, Andreu JM, Barasoain I. Taxanes: microtubule and centrosome targets, and cell cycle dependent mechanisms of action. Curr Cancer Drug Targets 2003; 3(3): 193-203. https://doi.org/10.2174/1568009033481967.

48. Semiglazov VF., Dashyan GA, Krivorotko PV, Paltuev RM, Donskikh RV, Semiglazova TYu, et al. New possibilities of the treatment of breast cancer: microtubule inhibitors. Farmateka 2017; (17(350)): 45-49. Russian. https://www.elibrary.ru/item.asp?id=30522619.

49. Howarth FC, Calaghan SC, Boyett MR, White E. Effect of the microtubule polymerizing agent taxol on contraction, $\mathrm{Ca} 2+$ transient and L-type Ca2+ current in rat ventricular myocytes. J Physiol 1999 516 (pt 2) (Pt 2): 409-419. https://doi.org/10.1111/j.14697793.1999.0409v.x.

50. Wang H, Cheng G, Du Y, Ye L, Chen W, Zhang L, et al. Hypersensitivity reaction studies of a polyethoxylated castor oil-free, liposome-based alternative paclitaxel formulation. Mol Med Rep 2013; 7(3): 947-952. https://doi.org/10.3892/mmr.2013.1264.

51. Ky B, Putt M, Sawaya H, French B, Januzzi JL Jr, Sebag IA, et al. Early increases in multiple biomarkers predict subsequent cardiotoxicity in patients with breast cancer treated with doxorubicin, taxanes, and trastuzumab. J Am Coll Cardiol 2014; 63(8): 809-816. https://doi.org/10.1016/j.jacc.2013.10.061.

52. Pentassuglia L, Timolati F, Seifriz F, Abudukadier K, Suter TM, Zuppinger $C$. Inhibition of ErbB2/neuregulin signaling augments paclitaxel-induced cardiotoxicity in adult ventricular myocytes. Exp Cell Res 2007; 313: 1588-1601. https://doi.org/10.1016/j.yexcr.2007.02.007.

53. Lluch A, Ojeda B, Colomer R, Barnadas A, Massuti B, Casado A, et al. Doxorubicin and paclitaxel in advanced breast carcinoma: importance of prior adjuvant anthracycline therapy. Cancer 2000; 89(11): 21692175. https://doi.org/10.1002/10970142(20001201)89:11\%3C2169::aid-cncr4\%3E3.0.co;2-9.

54. Mearini G, Schlossarek S, Willis MS, Carrier L. The ubiquitinproteasome system in cardiac dysfunction. Biochim Biophys Acta 2008 1782(12): 749-763. https://doi.org/10.1016/j.bbadis.2008.06.009.

55. Semochkin SV. New proteasome inhibitors in the management of multiple myeloma. Oncohematology 2019; 14(2): 29-40. Russian. https://doi.org/10.17650/1818-8346-2019-14-2-29-40.

56. Enrico O, Gabriele B, Nadia C, Sara G, Daniele V, Giulia C, et al. Unexpected cardiotoxicity in haematological bortezomib treated patients. $\mathrm{Br} J$ Haematol 2007; 138(3): 396-397. https://doi.org/10.1111/j.1365-2141.2007.06659.x.

57. Herndon TM, Deisseroth A, Kaminskas E, Kane RC, Koti KM, Rothmann MD, et al. U.s. Food and Drug Administration approval: carfilzomib for the treatment of multiple myeloma. Clin Cancer Res 2013; 19(17): 4559-4563. https://doi.org/10.1158/1078-0432.ccr-13-0755.

58. Nuver J, Smit AJ, van der Meer J, van den Berg MP, van der Graaf WT, Meinardi $\mathrm{MT}$, et al. Acute chemotherapy-induced cardiovascular changes in patients with testicular cancer. J Clin Oncol 2005; 23(36) 9130-9137. https://doi.org/10.1200/ico.2005.01.4092.

59. Feldman DR, Schaffer WL, Steingart RM. Late cardiovascular toxicity following chemotherapy for germ cell tumors. J Natl Compr Canc Netw 2012; 10(4): 537-544. https://doi.org/10.6004/incen.2012.0051.

60. Siddik ZH. Cisplatin: mode of cytotoxic action and molecular basis of resistance. Oncogene 2003; 22(47): 7265-7279. https://doi.org/10.1038/sj.onc.1206933.

61. Haugnes HS, Wethal T, Aass N, Dahl O, Klepp O, Langberg CW, et al. Cardiovascular risk factors and morbidity in long-term survivors of testicular cancer: a 20-year follow-up study. J Clin Oncol 2010; 28(30): 4649-4657. https://doi.org/10.1200/jco.2010.29.9362.

62. van den Belt-Dusebout AW, Nuver J, de Wit R, Gietema JA, ten Bokke Huinink WW, Rodrigus PT, et al. Long-term risk of cardiovascular 
disease in 5-year survivors of testicular cancer. J Clin Oncol 2006; 24(3): 467-475. https://doi.org/10.1200/jco.2005.02.7193.

63. Feldman DR, Jacobsen EP, Woo K, Steingart R, Fleisher M, Patil S, et al. Acute changes in endothelial function with cisplatin among germ cell tumor patients. J Clin Oncol 2014; 32(15_suppl): 9587-9587. https://doi.org/10.1200/jco.2014.32.15_suppl.9587.

64. Wachters FM, Van Der Graaf WT, Groen HJ. Cardiotoxicity in advanced nonsmall cell lung cancer patients treated with platinum and nonplatinum based combinations as first-line treatment. Anticancer Res 2004; 24(3b): 2079-2083. https://pubmed.ncbi.nlm.nih.gov/15274404.

65. Ylä-Herttuala S, Rissanen TT, Vajanto I, Hartikainen J. Vascular endothelial growth factors: biology and current status of clinical applications in cardiovascular medicine. J Am Coll Cardiol 2007; 49(10): 1015-1026. https://doi.org/10.1016/j.jacc.2006.09.053.

66. Motzer RJ, Hutson TE, Tomczak P, Michaelson MD, Bukowski RM, Rixe $O$, et al. Sunitinib versus interferon alfa in metastatic renal-cell carcinoma. N Engl J Med 2007; 356(2): 115-124. https://doi.org/10.1056/nejmoa065044.

67. Ferrara N. VEGF-A: a critical regulator of blood vessel growth. Eur Cytokine Netw 2009; 20(4): 158-163. https://doi.org/10.1684/ecn.2009.0170.

68. Horowitz JR, Rivard A, van der Zee R, Hariawala M, Sheriff DD, Esakof $\mathrm{DD}$, et al. Vascular endothelial growth factor/vascular permeability factor produces nitric oxide-dependent hypotension. Evidence for a maintenance role in quiescent adult endothelium. Arterioscler Thromb
Vasc
Biol 1997;
$17(11)$
2793-2800. https://doi.org/10.1161/01.atv.17.11.2793.

69. Eremina V, Sood M, Haigh J, Nagy A, Lajoie G, Ferrara N, et al. Glomerular-specific alterations of VEGF-A expression lead to distinct congenital and acquired renal diseases. I Clin Invest 2003; 111 (5): 707-716. https://doi.org/10.1172/ici17423.

70. Eremina V, Jefferson JA, Kowalewska J, Hochster $\mathrm{H}$, Haas M, Weisstuch J, et al. VEGF inhibition and renal thrombotic microangiopathy. $N$ Engl $\begin{array}{llll}\text { J Med } & \text { 2008; } & \text { 358(11): } & 1129-1136 .\end{array}$ https://doi.org/10.1056/nejmoa0707330.

71. Scappaticci FA, Skillings JR, Holden SN, Gerber HP, Miller K, Kabbinavar $F$, et al. Arterial thromboembolic events in patients with metastatic carcinoma treated with chemotherapy and bevacizumab. J Nat/ Cancer Inst 2007; 99(16): 1232-1239. https://doi.org/10.1093/inci/dim086.

72. Izumiya Y, Shiojima I, Sato K, Sawyer DB, Colucci WS, Walsh K. Vascular endothelial growth factor blockade promotes the transition from compensatory cardiac hypertrophy to failure in response to pressure overload. Hypertension 2006; 47(5): 887-893. https://doi.org/10.1161/01.hyp.0000215207.54689.31.

\section{Authors:}

Aleksey M. Chaulin - MD, Postgraduate student, Instructor, Department of Cardiology and Cardiovascular Surgery, Samara State Medical University, Samara, Russia; Physician, Samara Regional Clinical Cardiological Dispensary, Samara, Russia. https://orcid.org/0000-0002-2712-0227.

Olga E. Abashina - MD, Postgraduate student, Instructor, Department of Cardiology and Cardiovascular Surgery, Samara State Medical University, Samara, Russia. https://orcid.org/0000-0002-5302-6381.

Dmitry V. Duplyakov - MD, DSc, Professor, Department of Cardiology and Cardiovascular Surgery, Samara State Medical University, Samara, Russia; Deputy Chief Physician in Charge of Medical Matters, Samara Regional Clinical Cardiological Dispensary, Samara, Russia. https://orcid.org/00000002-6453-2976. 